\title{
A CASE OF TUBAL PREGNANCY,
}

\author{
WITH REMARKS ON THE CALSE OF EARLY \\ RUPTURE.
}

BY

\author{
J. BLAND SUT'TON, F.R.C.S., \\ ASSISTANT SURGFon to The MidDLESEX hospital.
}

Received October 1 th-Read November 12 th, 1889.

Ir would be superfluous to occupy the time of this Society with the details of a successful operation for ruptured tubal pregnancy, were it not that the case presents some exceptional features, and enables me to offer a few remarks on some points in the pathology of the accident.

Emma B-, æt. 37, came under my care in the Middlesex Hospital, August 26th, 1889. Patient has been twice married, her matrimonial life extends over a period of seventeen years. Has never been pregnant or suffered from gonorrhoea, and has always been regular with regard to the catamevia until three months before her admission, when she missed two periods. Five weeks before admission patient was seized with sudden violent pain in the abdonien. Dr. Clegg, of Stratford, was sent for, and on 
arriving found the woman collapsed. Slowly she reacted and refused to allow any vaginal examination to be made. In the course of a few days a swelling appeared on the right side of the abdomen. At the end of five weeks she was sent to me at the Middlesex Hospital.

On admission I found a swelling occupying the right iliac fossa, extending upwards to the costal arch and inwards as far as the middle line. The uterus was normal in position, and the sound entered three inches. The right side of Douglas's fossa was occupied by an ill-defined swelling, firm to the touch ; a rounded moveable nodule, of the bigness of a Tangerine orange, lay behind the uterus. No breast signs or history of vomiting. There was great tenderness over the abdominal aspect of the tumour. During the next twelve days the temperature ranged from $99^{\circ}$ in the morning to $101^{\circ}$ in the evening.

On September 6th (twelve days after admission) I opened the abdomen, and came upon a quantity of putrid, darkcoloured blood-clot filling the pelvis and right iliac fossa, and extending upwards to the liver. This was quickly removed, as well as the rounded moveable nodule in Douglas's fossa. As soon as the clot was turned out some smart bleeding came from the right broad ligament. This was quickly stopped by transfixing the ligament with a double silk ligature close to the uterus, afterwards cutting away the débris of the tube and ovary. 'The cavity of the pelvis and peritoneum were washed out with eight quarts of water at $110^{\circ}-115^{\circ}$. I then examined the left broad ligament, but the parts were so matted together that it was impossible to distinguish ovary or tube. The uterus was normal in size, shape, and position. A glass drainagetube was inserted and retained for three days. The temperature varied from $98^{\circ}$ to $99 \cdot 4^{\circ}$ during the six days following the operation, then rose somewhat as the track of the drainage-tube suppurated. The patient made an excellent recovery, due, I believe, to the use of the drainagetube.

On examining the parts removed I was able to recog- 
nise the remnants of an enlarged Fallopian tube, the ovary contained a corpus luteum of pregnancy. The rounded moveable mass consisted of what is kuown as an apoplectic ovum, and on washing the clot the cephalic extremity of an embryo was found, corresponding to the seventh or eighth week. An examination of the membranes is of interest, as it throws some light on the cause of these eariy ruptures in tubal pregnancies.

Obstetriciansare familiar with rounded masses discharged from the uterus of pregnant women accompanied by profuse hæmorrhage. Such rounded masses are known by a variety of names-blighted ovum, carneous mole, apoplectic ovum, cystic or tubercular ovum. They are so common that every pathological museum contains many specimens. In the middle of a blighted orum a cavity exists, usually lodging an ill-developed, misshapen embryo of about the fifth, sixth, or eighth week of pregnancy: occasionally only the stump of the cord is detected. A blighted or apoplectic ovum is an early embryo with its membranes, into which hæmorrhage has occurred. The extent of the extravasation varies; sometimes the whole of the membranes are infiltrated, and occasionally the blood invades the amniotic cavity and overwhelms the embryo.

The specimen I show to-night is an apoplectic ovum from the Fallopian tube, and a glance at the drawing (Fig. 1) will be sufficient to establish its identity. This is the key, I think, to some of these early ruptures in tubal pregnancy. For instance an ovum (using this term to include an embryo and its membranes) the size of a walnut is suddenly enlarged to the size of an orange by hæmorrhage into its membranes. When lodged in the uterus this event causes sufficient disturbance to bring about expulsion of the ovum, accompanied by free bleeding: in the Fallopian tube this accident produces rupture, with discharge of the ovum into the peritoneal cavity, accompanied by profuse hæmorrhage; sometimes the extravasation takes place into the broad ligament, but in early cases this appears to be uncommon. 
I do not base this opinion on one case. A few weeks ago I made a report on a similar specimen, and as the details

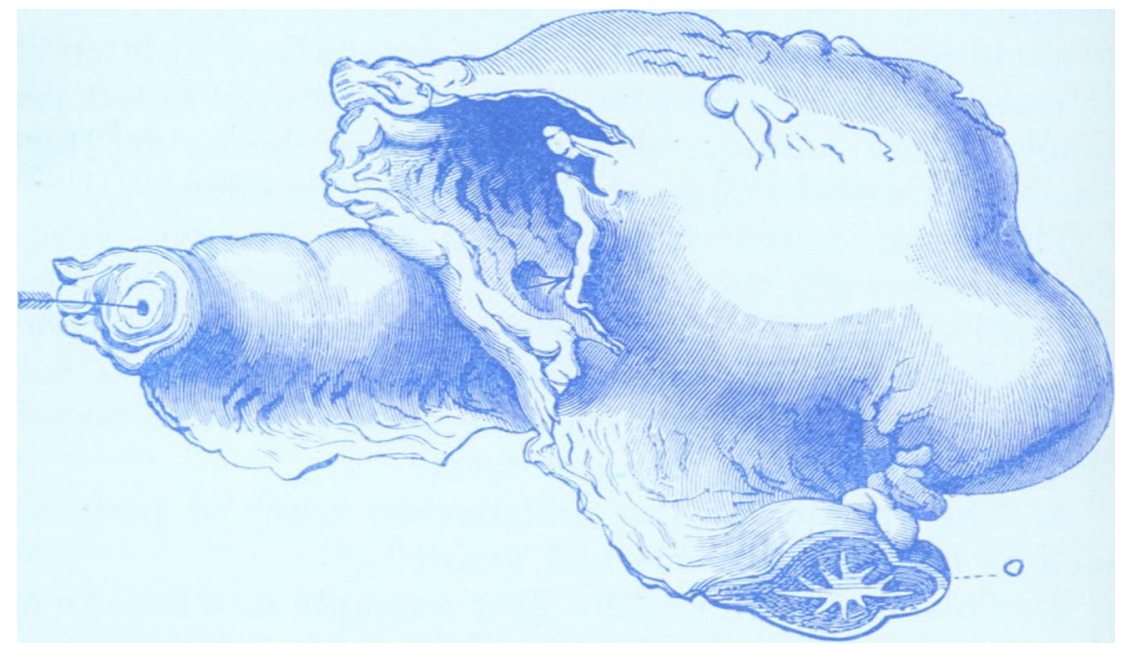

Fig. 1.-The distended Fallopian tube showing the situation of the rupture; o, ovary with corpus luteum.

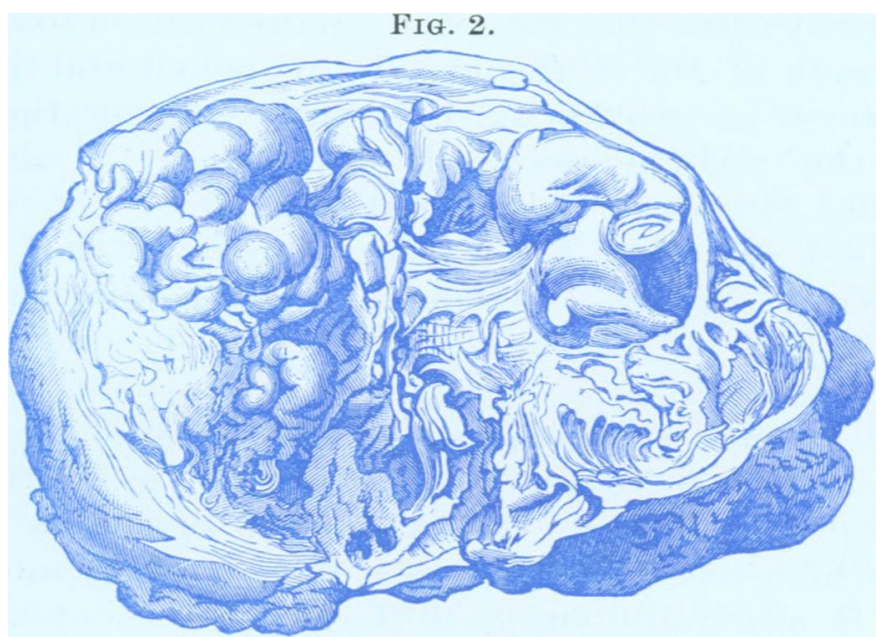

Fig. 3.

Fra. 2. - Apoplectic ovum from the Fallopian tube, represented in Fig. 1. FIG. 3.- Distorted head of the fœtus. About seventh or eighth week of gestation.

of the case will probably be published shortly, further remarks upon it must not come from me. 
Specimens of intra-peritoneal hæmatocele, as they are called, have been recorded and shown at societies, as examples of ruptured tubal pregnancies, but no embryo or membranes were found. I am strongly of opinion that no case should be regarded as due to ruptured tubal pregnancy unless membranes, or fotus, or both, are forthcoming, however suggestive the clinical evidence.

The most noteworthy clinical facts in the case of Emma B- were these :

1. She had not been pregnant previously, although married seventeen years, yet the first pregnancy was tubal.

2. The rupture, though intra-peritoneal and accompanied by profuse bleeding, was not fatal.

3. The absence of the conspicuous signs of pregnancy such as enlarged breasts and vomiting.

4. This is, I believe, the first example in which an apoplectic ovum has been recorded as occurring in the Fallopian tube.

Note.-Shortly after this case was communicated to the Society the track of the drainage-tube reopened and discharged pus for a few weeks, until the three silk ligatures with which the pedicle was tied came away; it then closed, and has given rise to no further trouble.

May 23rd.-I saw Mrs. B- to-day, and found her in the best of health, and able to attend to household duties as formerly.

Addendum.- Since this paper was written and placed in the hands of the secretary I have, with the aid of the light it appears to furnish, re-examined a specimen of hæmatocele which has, I regret to say, been wrongly interpreted. As it admirably supports the contention of this paper I will briefly describe it.

Alice $\mathrm{H}-$, æt. 25, came under the care of my colleague Dr. W. Duncan in August, 1886. She was married, had three children, the youngest being, at the time of her admission, two years old. Since the last confinement the 
patient had suffered from pelvic pain and painful menorrhagia. * Vaginal examination revealed an elastic swelling the size of a Tangerine orange to the left of the uterus. The swelling was regarded as a dilated Fallopian tube. In September, 1886, after consulting with my colleagues, $I$ opened the abdomen and removed the uterine appendages. The left ovary was adherent to a fold of omentum which contained coagulated blood, and constituted the swelling which could be felt by the vagina. The ovaries were cystic, and the hæmorrhage was attributed to rupture of one of the enlarged follicles. The patient made an admirable recovery, and $I$ preserved the hæmatocele as a pathological curiosity. My interest in the matter induced me to re-examine the specimen, with the following result :

One inch from the abdominal ostium of the Fallopian tube there is a rupture exposing for some distance the mucous membrane of the tube. Close beside this, embedded in laminated clot, is an apoplectic ovum of the bigness of a chestnut, and a few delicate fringes project from it. These, when examined microscopically, show the dendritic arrangement of the villi of the chorion. The hæmatocele in this case was not due to the rupture of a follicle, but to a ruptured tubal pregnancy of very early date (probably fourth or fifth week). It is the smallest apoplectic ovum I have as yet examined. From the history it is impossible to decide the date of the accident, but the hæmatocele had been noticed for many weeks previously to the operation, and there was no evidence of peritonitis beyond the few adhesions between the ovary, tube, and the omentum which iumediately encysted the bloodclot.

(For report of the discussion on this paper, see 'Proceedings of the Royal Medical and Chirurgical Society,' Third Series, vol. ii, p. 18.) 